\title{
The Effects of Initial Procalcitonin Levels on Mortality Rates in Geriatric Patients Undergoing Surgery
}

Belkiz Ongen İpek ${ }^{1}$, Aslı Karadeniz ${ }^{2}$, Mustafa Erinc Sitar ${ }^{3}$

1. Medical Biochemistry, Maltepe University Faculty of Medicine, İstanbul, TUR 2. Infectious Disease, Maltepe University, Istanbul, TUR 3. Medical Biochemistry, Maltepe University Faculty of Medicine, Istanbul, TUR

Corresponding author: Mustafa Erinc Sitar, merincsitar@maltepe.edu.tr

\section{Abstract \\ Introduction}

The aim of the current study is to investigate the relationship between mortality rate in geriatric patients undergoing surgery with preoperative serum levels of procalcitonin, C-reactive protein, and erythrocyte sedimentation rate.

\section{Methods}

This was a single-center retrospective study, including three groups with 101 patients, who are older than 65 years of age. A retrospective investigation was carried out from the laboratory information system for all groups from January to December 2018. Group 1 included patients who had surgery and then mortality within 30 days after surgery. Group 2 included hospitalized patients who had surgery and no mortality within 30 days after surgery. Group 3 included outpatient patients, who had suspicion for a bacterial infection and then no surgery or no mortality within 30 days.

\section{Results}

When three group comparisons were made for procalcitonin, C-reactive protein, and erythrocyte sedimentation rate values, the p-value of one-way analysis of variance (ANOVA) was higher than 0.05 for procalcitonin and lower than 0.05 for C-reactive protein and erythrocyte sedimentation rate, suggesting that one or more groups were significantly different. When post-hoc multiple comparison methods were applied, there were statistically significant differences between Groups 1 and 3 for C-reactive protein and erythrocyte sedimentation rate.

\section{Conclusions}

Procalcitonin levels do not predict mortality following surgery. C-reactive protein and erythrocyte sedimentation rate are more useful biomarkers predicting mortality in geriatric patients undergoing surgery.

Received 03/19/2020 Review began 03/23/2020 Review ended 03/26/2020 Published 04/10/2020

\section{() Copyright 2020}

Ongen Ipek et al. This is an open access article distributed under the terms of the Creative Commons Attribution License CC-BY 4.0., which permits unrestricted use, distribution, and reproduction in any medium, provided the original author and source are credited.
Categories: Internal Medicine, Infectious Disease

Keywords: geriatric medicine, mortality, procalcitonin, surgery

\section{Introduction}

According to a World Health Organization (WHO) report, aging is defined as a process that progresses with the accumulation of gradual damage at the molecular and cellular levels and continues with a reduction in physiological and cognitive capacity, eventually resulting in inevitable death [1]. Although life span has not changed in the present century, life expectancy is increasing day by day and the average life expectancy in today's average world society is over 60 years old [2]. High success rates in vaccination, increased access to antibiotic use, rapid access to the health system, effective communication and logistic systems, and increased success rates in pharmacological treatments of chronic diseases are positively reflected in these statistics. As the age of the population increases gradually, the prevalence of diseases such as Alzheimer's, Parkinson's, malignancies, Type 2 diabetes mellitus, cataracts, osteoporosis, and hypertension is increasing. Infections should be included in this list as well. The elderly population has higher numbers of ongoing challenges due to atypical and/or ambiguous clinical presentations as compared to younger peers. In addition, they have increased comorbidity and death risk together with low immunological status [3]. It is crucial to identify geriatric patients who have an increased risk of mortality and develop strategies to prevent the outcome. Severity status grading systems, which are routinely applied for critically ill people like Acute Physiologic Assessment and Chronic Health Evaluation (APACHE) II, Mortality Prediction Model (MPM), Sequential Organ Failure Assessment (SOFA), and Simplified Acute Physiology Score (SAPS II) may have irrelevant scores in elderly and/or septic patients. If patients at risk for sepsis and mortality are detected more quickly, there may be a chance to take precautions and reduce mortality rates. In this fundamental problem, medical laboratories can contribute to clinical practice with biomarkers that mirror 
Procalcitonin (ProCT) entered the scientific literature in the 1970s [4]. It was found out to be a higher molecular weight precursor of calcitonin. In the early 90s, it has been found out that it has a linear relation with microbial invasion and gives information for the differentiation of viral infections from bacterial ones [5]. In current practice, ProCT gives valuable data in the primary diagnosis of bacterial sepsis and guide antibiotic therapy [6-8]. It also assists the clinician in the selection of pharmacological agents, monitoring the effective response to treatment and duration of administration. For critical reasons such as these, the use of ProCT is increasing day by day all over the world. However, more detailed assessments of the performance of the ProCT test in geriatric patients are required. Actually, when the clinician suspects a severe bacterial infection, ProCT levels can enlighten the course. However, the situation may not be so simple in elderly patients [9]. In the literature, there are publications with different results for the relationship between ProCT and mortality. Higashikawa et al. investigated ProCT levels in the geriatric population with suspected bacterial infection [10]. They found that 30-day mortality was importantly elevated with PCT levels $>0.5 \mathrm{ng} / \mathrm{mL}$. On the other hand, Szakmary and Molnar revealed different data in their study [11]. They stated that ProCT levels do not yield enough clinical information relevant to mortality rates after major operations. Similar findings also exist for other important markers such as erythrocyte sedimentation rate (ESR) and C-reactive protein (CRP). It is well-known that CRP is a serum biomarker of an acute-phase reaction and can be elevated in inflammation, bacterial infection, and trauma [12-13]. But there is a lack of strong evidence about the prognostic value of serum biomarkers after surgery in elderly people. The aim of the current study is to investigate the relationship between mortality rate in geriatric patients undergoing surgery with the preoperative serum levels of ProCT, CRP, and ESR.

\section{Materials And Methods}

This study was approved by the local clinical ethics board of Maltepe University Faculty of Medicine, Istanbul (issue number 2019/900/15). We conducted a retrospective study including three groups - 101 patients, who are older than 65 years of age, admitted to Maltepe University Educational and Research Hospital, Istanbul. The retrospective investigation was accomplished from the laboratory information system for all groups from January to December 2018. Group 1 included patients who had surgery and then mortality within 30 days after surgery $(n=28)$. Group 2 included hospitalized patients who had surgery and no mortality within 30 days after surgery. Group 3 included outpatient patients who had suspicion of bacterial infection and then no surgery or no mortality within 30 days (Figure 1). Phlebotomies were applied on the first day of admission between 8 am to 10 am after overnight fasting according to the standard venipuncture quality procedures of the hospital. All samples were centrifuged after venous blood sampling. Serum ProCT levels were measured by Roche Hitachi E170 (Basel, Switzerland) using the immunoassay method after centrifugation. Blood urea nitrogen (BUN), creatinine, albumin, total protein, aspartate aminotransferase (AST), and alanine aminotransferase (ALT) activity levels were measured by Siemens (Munich, Germany) Dimension RXLmax after centrifugation according to the instructions of the manufacturer. The sedimentation rate was analyzed by Electalab (Forli Italy) Sedy40 and complete blood count was measured by Sysmex (Kobe Japon) XT2000i.

\section{Group 1}

Hospitalized patients $\mathrm{N}=28$

$>65$ age

$\downarrow$

Initial ProCT measurement

$\downarrow$

Surgery

$\downarrow$

Mortality within 30 days
Group 2

Hospitalized patients $\mathrm{N}=37$

$>65$ age

$\downarrow$

Initial ProCT measurement

$\downarrow$

Surgery

$\downarrow$

No mortality within 30 days
Group 3

Outpatient patients with risk of bacterial infection $\mathrm{N}=36$ $>65$ age $\downarrow$

Initial ProCT measurement $\downarrow$

No surgery

$\downarrow$ No mortality within 30 days

FIGURE 1: Flow chart of patients recruited in the present study (ProCT)

ProCT: procalcitonin

Biostatistical analyses were performed using SPSS version 21 (SPSS Inc., Chicago, Illinois) and p-value $<0.05$ was considered statistically significant. The comparison between the groups in the parametric results was made by analysis of variance (ANOVA. When there was a difference between the groups, post-hoc multiple comparison methods Tukey, Bonferroni, and Scheffé tests were used to determine which group caused the difference. 


\section{Cureus}

\section{Results}

A total of 101 geriatric patients were included in the study; the mean age of patients was demonstrated in Figure 2 (higher than 80 years for the entire study group). ProCT mean \pm SD values were found to be $7.2 \pm 21$ for Group 1, 3.93 \pm 13 for Group 2, and $0.58 \pm 1.2$ for Group 3. CRP mean \pm SD values were obtained as $9.4 \pm 9$ for Group 1, 7.3 \pm 7 for Group 2, and $5.5 \pm 5$ for Group 3. ESR mean \pm SD values were obtained as $63 \pm 22$ for Group 1 , $51 \pm 26$ for Group 2, and 44 \pm 22 for Group 3 (Table 1). The groups did not differ in ProCT levels $[\mathrm{F}(2,113)=2.37$, $p=0,0983]$. Significant differences were found for $\operatorname{CRP}[F(2,104)=3.30, p=0,0409]$ and ESR levels $[F(2$, $104)=5.58, \mathrm{p}=0.0050]$, indicating significant difference among groups. Tukey's post-hoc test demonstrated significantly lower CRP and ESR levels in Group 3 in comparison to Group 1 ( $\mathrm{p}<0.05$ and $\mathrm{p}<0.001$, respectively) (Table 2). Biochemical and immunoassay parameters of all groups were shown in Table 1 together with neutrophil, lymphocyte, thrombocyte counts, blood urea nitrogen (BUN), creatinine, AST, ALT, albumin, ProCT, CRP, and ESR.

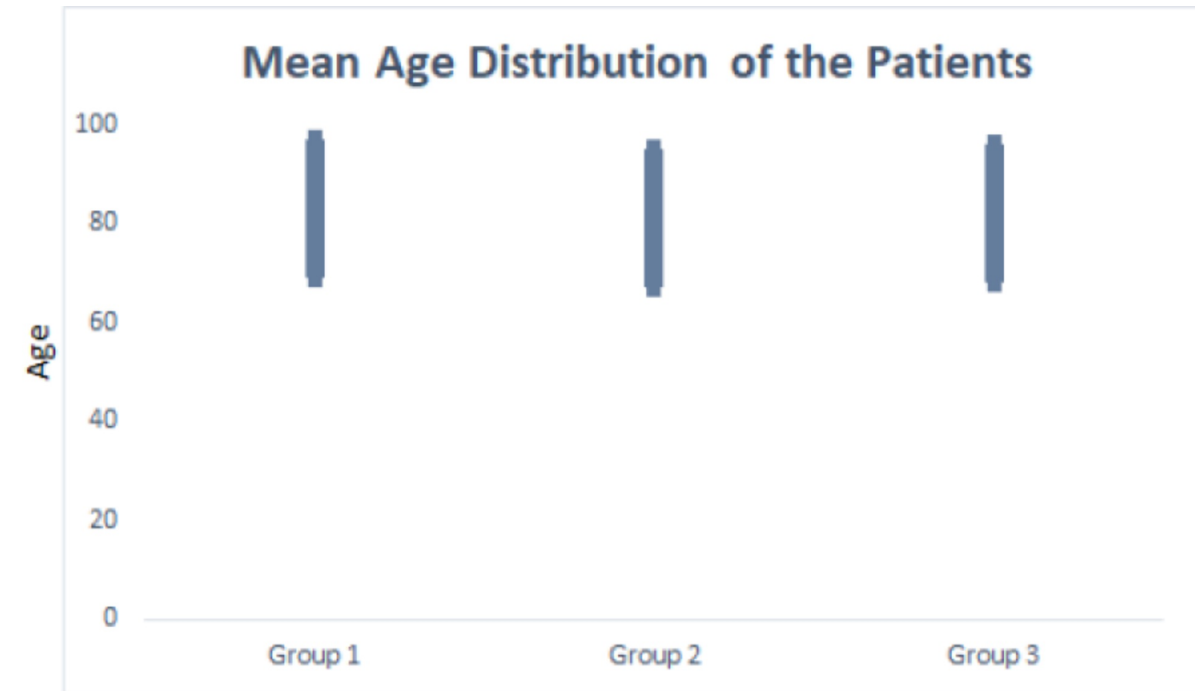

FIGURE 2: Mean ages of the groups and standard deviations

\begin{tabular}{|c|c|c|c|}
\hline & Group 1 (Mean $\pm S D)$ & Group $2($ Mean $\pm S D)$ & Group $3($ Mean $\pm S D)$ \\
\hline BUN (mg/dL) & $52,17 \pm 41$ & $28 \pm 17$ & $40,73 \pm 34$ \\
\hline Creatinine (mg/dL) & $1,74 \pm 1,3$ & $1,01 \pm 0,6$ & $1,65 \pm 1$ \\
\hline AST (U/L) & $63,3 \pm 82$ & $26,97 \pm 18,7$ & $80,46 \pm 284,6$ \\
\hline ALT (U/L) & $51,5 \pm 68$ & $23,8 \pm 11$ & $69,21 \pm 181,5$ \\
\hline Albumin (mg/dL) & $2,37 \pm 0,6$ & $2,61 \pm 0,5$ & $2,9 \pm 0,6$ \\
\hline Neutrophil $\left(10^{3} / \mu \mathrm{L}\right)$ & $9,08 \pm 6$ & $8,6 \pm 4,8$ & $6,8 \pm 4$ \\
\hline Lymphocyte $\left(10^{3} / \mu \mathrm{L}\right)$ & $1,3 \pm 8$ & $1,5 \pm 7$ & $1,4 \pm 6$ \\
\hline Thrombocyte $\left(10^{3} / \mu \mathrm{L}\right)$ & $212,3 \pm 112$ & $212,2 \pm 94$ & $243,2 \pm 107$ \\
\hline ProCT ( $\mu \mathrm{g} / \mathrm{L})$ & $7,2 \pm 21$ & $3,93 \pm 13$ & $0,58 \pm 1,2$ \\
\hline ESR & $63 \pm 22$ & $51 \pm 26$ & $44 \pm 22$ \\
\hline $\mathrm{CRP}(\mathrm{mg} / \mathrm{L})$ & $9,4 \pm 9$ & $7,3 \pm 7$ & $5,5 \pm 5$ \\
\hline
\end{tabular}

TABLE 1: Biochemical parameters of all groups together with neutrophil, lymphocyte, thrombocyte counts, BUN, creatinine, AST, ALT, albumin, ProCT, ESR, and CRP

BUN: blood urea nitrogen; AST: aspartate aminotransferase; ALT: alanine aminotransferase; ProCT: procalcitonin; ESR: erythrocyte sedimentation rate; CRP: C-reactive protein 


\section{Cureus}

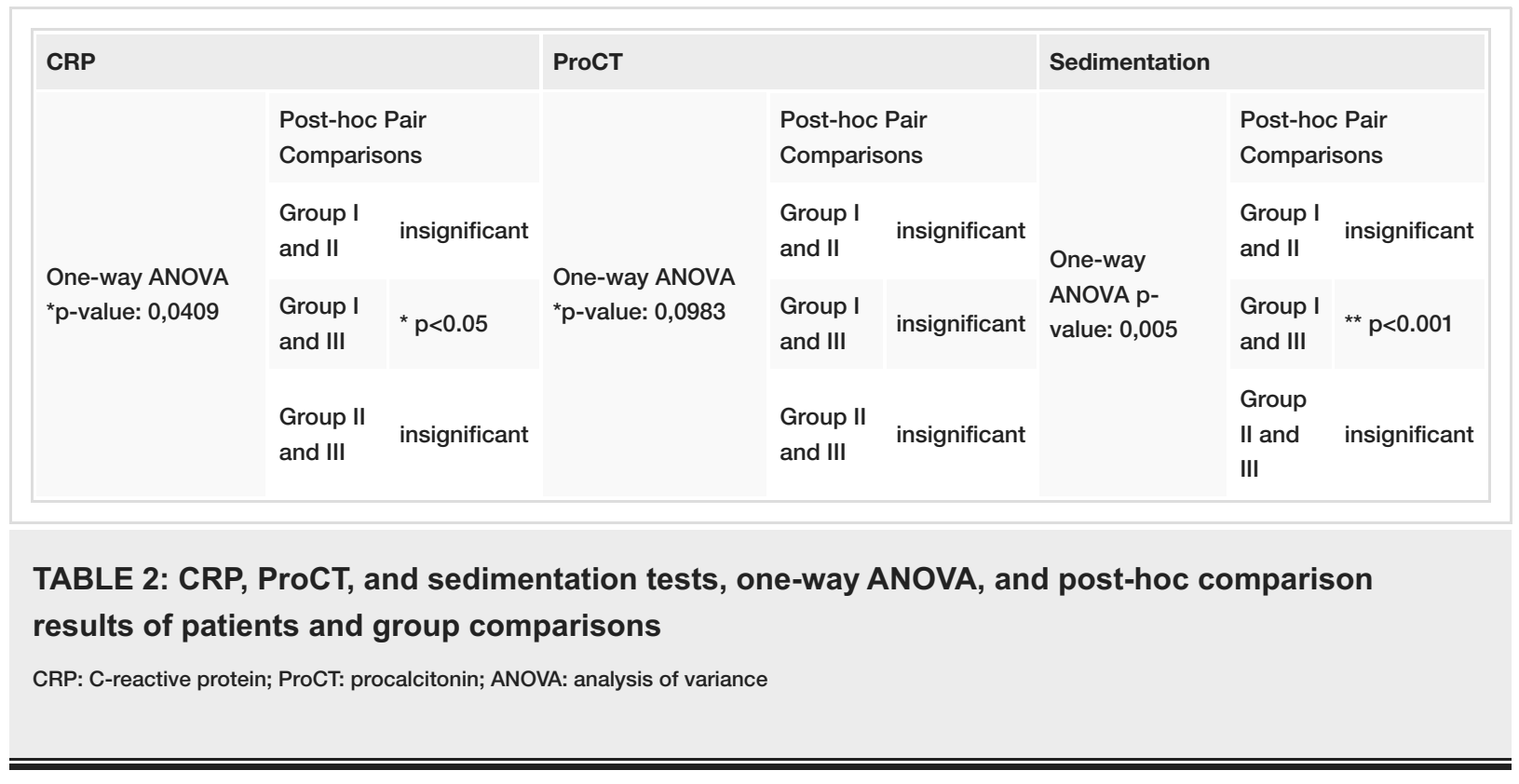

\section{Discussion}

Our study indicated that ProCT levels did not predict mortality following surgery, however, CRP and ESR were more useful biomarkers predicting mortality in geriatric patients undergoing surgery.

There are distinctive studies about ProCT in the elderly population and mortality predictions in the current medical literature. Yu H et al. searched the levels of ProCT and the 30-day mortality rate, together with a quick sequential organ failure assessment (SOFA) score, in hospitalized patients. They found a significant correlation between those parameters unlike our findings [14]. Fritz et al. assessed ProCT levels and mortality after cardiopulmonary bypass surgery [15]. They concluded that elevated levels of ProCT (>2.5 ngl1) are predictive of 28-day mortality. On the contrary, Szakmany stated that ProCT levels do not predict mortality following major abdominal surgery [11]. This study contains findings consistent with our study. Clementi et al. studied presepsin and ProCT levels for mortality in cardiac surgery patients [16]. They found that the predictive value for in-hospital, 30-day, and six-month mortality was higher for presepsin. Kim et al. found that ProCT levels were associated with pneumonia intensity but not with mortality in elderly patients with community-acquired pneumonia [17]. Vallet $\mathrm{H}$ et al showed a significant association of high ProCT values with mortality within 30 days for geriatric patients after orthopedic surgery [18]. Stucker et al. investigated ProCT in detecting infection in elderly patients [19]. They concluded that increased ProCT levels $>0.5 \mathrm{ng} / \mathrm{mL}$ is associated with infection in the geriatric population. Lee et al. studied ProCT levels with bacterial infections in the geriatric population [20]. They concluded that ProCT can be used to exclude sepsis in the geriatric population. In our study, we found that ProCT levels were not giving information about onemonth mortality properly.

Increased serum CRP levels are independently determinative for elevated mortality and poor clinical outcome in elderly patients with vascular diseases such as atherosclerosis, coronary heart disease and stroke [21-23]. Kim et al. investigated preoperative CRP levels as a one-year mortality predictor in elderly patients who underwent hip fracture surgery [24]. They found that one-year mortality was significantly increased in patients whose CRP levels were higher than $10 \mathrm{mg} / \mathrm{dL}$. All these results correlate very well with the current study. On the other hand, Beloosesky et al. found no association between CRP levels and six-month mortality in geriatric patients who underwent hip-fracture surgery [25]. These controversial findings raise a question about the predictive value of CRP.

ESR is the measurement of red blood cell aggregation, which rises when an augmentation in the total mass exceeds the increase in volume [26]. ESR is a biomarker of inflammation and can be elevated in rheumatoid arthritis, Crohn's disease, ulcerative colitis, and cancer [27]. Shibuya et al. searched the relationship between ESR and the postoperative mortality of patients with colorectal cancer [27]. They found that preoperative high levels of ESR (>40 mm/h) can predict poorer postoperative survival in patients with colorectal cancer. Seong et al. investigated the relationship between inflammatory biomarkers such as CRP, ESR, white blood cell count (WBC), neutrophil/ lymphocyte ratio and postoperative survival of patients with colorectal cancer [26]. They concluded that CRP, ESR, and the neutrophil/lymphocyte ratio were significantly associated with survival relevant to the current study.

The main limitations of our study are that it is a single-center study, with a variety of operations, and ProCT relevance to bacterial infections rather than viral ones. However, if the mean age of patients is considered, this study is highly valuable to guide future studies. 


\section{Conclusions}

It is necessary to focus on "healthy aging" rather than preventing or stopping aging. Because clinical laboratories contribute more than $70 \%$ to clinical processes, they must assume their role in healthy aging as well. Prediction of bad outcomes can be very beneficial for both health care professionals and the elderly themselves. We suggest day-by-day measurement of ProCT values for every geriatric patient and manufacturing a systematic panel using other grading systems. And it should always be kept in mind that every geriatric population is special clinically.

\section{Additional Information \\ Disclosures}

Human subjects: Consent was obtained by all participants in this study. Ethical committee of Maltepe University Medical Faculty issued approval 2019/900/15. Animal subjects: All authors have confirmed that this study did not involve animal subjects or tissue. Conflicts of interest: In compliance with the ICMJE uniform disclosure form, all authors declare the following: Payment/services info: All authors have declared that no financial support was received from any organization for the submitted work. Financial relationships: All authors have declared that they have no financial relationships at present or within the previous three years with any organizations that might have an interest in the submitted work. Other relationships: All authors have declared that there are no other relationships or activities that could appear to have influenced the submitted work.

\section{Acknowledgements}

We deeply thank our experienced laboratory technicians, Esra Gulnaroglu and Hatice Ejderoglu, for their robust measurements of ProCT in our central laboratory.

\section{References}

1. World Health Organization: World Report on Ageing and Health. World Health Organization, Luxemburg; 2015.

2. World Economic and Social Survey 2007: Development in an Aging World . (2007). https://www.un.org/development/desa/ageing/publications/2008/08/world-economic-and-social-survey2007-development-in-....

3. Sitar ME, Alasehir EA, Ipek OB: Immune Modulation and Its Role in Antiaging. Molecular Basis and Emerging Strategies for Anti-aging Interventions. Rizvi SI, Çakatay U (ed): Springer, Singapore; 2018. 111132. 10.1007/978-981-13-1699-9

4. Roos BA, Okano K, Deftos LJ: Evidence for a pro-calcitonin. Biochem Biophys Res Commun. 1974, 60:11341140. 10.1016/0006-291x(74)90430-6

5. Assicot M, Gendrel D, Carsin H, J. Raymond, Carsin H, Guilbaud J: High serum procalcitonin concentrations in patients with sepsis and infection. Lancet. 1993, 341:515-518. 10.1016/0140-6736(93)90277-n

6. Meisner M: Update on procalcitonin measurements. Ann Lab Med. 2014, 34:263-273. 10.3343/alm.2014.34.4.263

7. Eddy F, Joyce A, Dryden M, et al.: Procalcitonin in older patients; promoting antibiotic stewardship in complex patients. J Infect Non Infect Dis. 2015, [Epub ahead of print]: 10.24966/INID-8654/100002

8. Sitar ME, Ipek BO, Karadeniz A: Procalcitonin in the diagnosis of sepsis and correlations with upcoming novel diagnostic markers. Int J Med Biochem. 2019, 2:132-140. 10.14744/ijmb.2019.30502

9. Lee WJ, Woo SH, Kim DH, et al.: Are prognostic scores and biomarkers such as procalcitonin the appropriate prognostic precursors for elderly patients with sepsis in the emergency department?. Aging Clin Exp Res. 2016, 28:917-924. 10.1007/s40520-015-0500-7

10. Higashikawa T, Okuro M, Ishigami K, et al.: Procalcitonin and albumin as prognostic biomarkers in elderly patients with a risk of bacterial infection. J Int Med Res. 2018, 46:2606-2614. 10.1177/0300060518766640

11. Szakmany T, Molnar Z: Procalcitonin levels do not predict mortality following major abdominal surgery . Can J Anaesth. 2003, 50:1082-1083. 10.1007/BF03018387

12. Gebhard F, Pfetsch H, Steinbach G, Strecker W, Kinzl L, Brückner UW: Is interleukin 6 an early marker of injury severity following major trauma in humans?. Arch Surg. 2000, 135:291-295. 10.1001/archsurg.135.3.291

13. Scherer MA, Neumaier M, von Gumppenberg S: C-reactive protein in patients who had operative fracture treatment. Clin Orthop Relat Res. 2001, 393:287-293. 10.1097/00003086-200112000-00033

14. Yu H, Nie L, Liu A, et al.: Combining procalcitonin with the qSOFA and sepsis mortality prediction . Medicine. 2019, 98:e15981. 10.1097/MD.0000000000015981

15. Fritz H, Brandes H, Bredle D, et al.: Post-operative hypoalbuminaemia and procalcitonin elevation for prediction of outcome in cardiopulmonary bypass surgery. Acta Anaesthesiol Scand. 2003, 47:1276-1283. 10.1046/j.1399-6576.2003.00239.x

16. Clementi A, Virzi GM, Mucino-Bermejo MJ, et al.: Presepsin and procalcitonin levels as markers of adverse postoperative complications and mortality in cardiac surgery patients. Blood Purif. 2019, 47:140-148. 10.1159/000494207

17. Kim JH, Seo JW, Mok JH, et al.: Usefulness of plasma procalcitonin to predict severity in elderly patients with community-acquired pneumonia. Tuberc Respir Dis (Seoul). 2013, 74:207-214. 10.4046/trd.2013.74.5.207

18. Vallet H, Chenevier-Gobeaux C, Villain C, et al.: Prognostic value of serum procalcitonin after orthopedic surgery in the elderly population. J Gerontol A Biol Sci Med Sci. 2017, 72:438-443. 10.1093/gerona/glw097 


\section{Cureus}

19. Stucker F, Herrmann F, Graf JD, Michel J-P, Krause KH, Gavazzi G: Procalcitonin and infection in elderly patients. J Am Geriatr Soc. 2005, 53:1392-1395. 10.1111/j.1532-5415.2005.53421.x

20. Lee SH, Chan RC, Wu JY, Chen HW, Chang SS, Lee CC: Diagnostic value of procalcitonin for bacterial infection in elderly patients-a systemic review and meta-analysis. Int J Clin Pract. 2013, 67:1350-1357. 10.1111/ijcp.12278

21. Buckley DI, Fu R, Freeman M, Rogers K, Helfand M: C-reactive protein as a risk factor for coronary heart disease: a systematic review and meta-analyses for the U.S. Preventive Services Task Force. Ann Intern Med. 2009, 151:483-495. 10.7326/0003-4819-151-7-200910060-00009

22. Nordestgaard BG, Zacho J: Lipids, atherosclerosis and CVD risk: is CRP an innocent bystander? . Nutr Metab Cardiovasc Dis. 2009, 19:521-524. 10.1016/j.numecd.2009.07.005

23. Elkind MS, Luna JM, Moon YP, Liu KM, Spitalnik SL, Paik MC, Sacco RL: High-sensitivity C-reactive protein predicts mortality but not stroke: the Northern Manhattan Study. Neurology. 2009, 73:1300-1307.

10.1212/WNL.0b013e3181bd10bc

24. Kim BG, Lee YK, Park HP, et al.: C-reactive protein is an independent predictor for 1-year mortality in elderly patients undergoing hip fracture surgery: a retrospective analysis. Medicine. 2016, 95:e5152. 10.1097/MD.0000000000005152

25. Beloosesky Y, Grinblat J, Pirotsky A, Weiss A, Hendel D: Different C-reactive protein kinetics in postoperative hip-fractured geriatric patients with and without complications. Gerontology. 2004, 50:216-222. 10.1159/000078350

26. Seong MK: Prognostic inflammation score in surgical patients with colorectal cancer . J Korean Med Sci. 2015, 30:1793-1799. 10.3346/jkms.2015.30.12.1793

27. Shibuya N, Ishizuka M, Takagi K, Iwasaki Y, Hachiya H, Aoki T, Kubota K: Relationship between preoperative erythrocyte sedimentation rate and survival after surgery in patients with colorectal cancer. Anticancer Res. 2018, 38:6783-6788. 10.21873/anticanres.13049 